\title{
Non-Scattering of the Solution of the Nonlinear Schrödinger Equation on the Torus
}

\author{
Xing Cheng, Qiang Li \\ College of Science, Hohai University, Nanjing, China \\ Email: chengx@hhu.edu.cn
}

How to cite this paper: Cheng, $\mathrm{X}$. and $\mathrm{Li}$, Q. (2017) Non-Scattering of the Solution of the Nonlinear Schrödinger Equation on the Torus. Journal of Applied Mathematics and Physics, 5, 1917-1922. https://doi.org/10.4236/jamp.2017.59162

Received: September 8, 2017 Accepted: October 13, 2017 Published: October 16, 2017

\begin{abstract}
In this article, we will show non-scattering of the solution of the nonlinear Schrodinger equation on the torus. The result extends the result of Colliander, J., Keel, M., Staffilani, G., Takaoka, H. and Tao, T. for the cubic nonlinear Schrödinger equation on 2-dimensional torus.
\end{abstract}

\section{Keywords}

Schrödinger Equation, Torus, Scattering

\section{Introduction}

In this article, we will consider the nonlinear Schrödinger equation on d-dimensional torus:

$$
\left\{\begin{array}{c}
i \partial_{t} u+\Delta u=|u|^{p-1} u . \\
u(0, x)=u_{0}(x) \in H^{1}\left(\mathbb{T}^{d}\right),
\end{array}\right.
$$

where $u$ : $\mathbb{R} \times \mathbb{T}^{d} \rightarrow \mathbb{C}, 1<p<\infty$. This kind of nonlinear Schrödinger equations has been studied intensively in the last two decades. See [1] [2] [3] [4].

Many mathematicians believe this equation does not have nontrivial solutions which scatter, i.e. which approach a solution to the linear equation at time $t=$ $\infty$. Colliander, J., Keel, M., Staffilani, G., Takaoka, H. and Tao, T. [5] consider the cubic nonlinear Schrödinger equation on two dimensional torus, and prove the solution cannot scatter to free solution in $H^{1}\left(\mathbb{T}^{d}\right)$.

As in [5], the explicit solution

$$
u(t, x)=\mathrm{Ae}^{i k} e^{i n x} e^{i t|n|^{2}} e^{i|A|^{p-1} t},
$$

where $A \geq 0, k \in \frac{\mathbb{R}}{2 \pi \mathbb{Z}}, n \in \mathbb{Z}^{d}$ cannot converge to a free solution $\mathrm{e}^{i t \Delta} \mathrm{u}_{+}$due to the presence of the phase rotation $\mathrm{e}^{i|A|^{p-1} t}$ which is caused by the nonlinearity. We will show this is the only solution that scatters modulo phase rotation in $\mathrm{H}^{1}$ 
in the sense that there exists $u_{+} \in H^{1}\left(\mathbb{T}^{d}\right)$ and function $\theta: \mathbb{R} \rightarrow \mathbb{R} / 2 \pi \mathbb{Z}$ such that

$$
\left\|u(t)-e^{i \theta(t)} e^{-i t \Delta} u_{+}\right\|_{H^{1}\left(\mathbb{T}^{d}\right)} \rightarrow 0 \text {, as } t \rightarrow \infty .
$$

is of the form (2), which then reveals that no solution of the nonlinear Schrödinger equation on torus can scatter to free solution.

\section{Main Theorem}

In this section, we will present the main theorem in this article. We will show the only solution that scatters modulo phase rotation is of the form (2).

Theorem 1 (No non-trivial solution scatters modulo phase rotation). Let $u: \mathbb{R} \times \mathbb{T}^{d} \rightarrow \mathbb{C}$ be an $\mathrm{H}^{1}$ solution to (1) which scatters modulo phase in $\mathrm{H}^{1}$, then $\mathrm{u}$ is of the form (2) for some $A \geq 0, k \in \mathbb{R} / 2 \pi \mathbb{Z}, n \in \mathbb{Z}^{d}$.

To prove this theorem, we first need some lemmas.

Lemma 2 (Pre-compactness). For $u_{+} \in \mathrm{H}^{1}\left(\mathbb{T}^{d}\right),\left\{e^{i \theta} e^{i t \Delta} u_{+}: \theta \in\right.$ $\mathbb{R} / 2 \pi \mathbb{Z}, t \in \mathbb{R}\}$ is pre-compact in $H^{1}\left(\mathbb{T}^{d}\right)$.

Proof. It is equivalent to show $\left\{e^{i \theta} e^{-i t|n|^{2}} \hat{u}_{+}(n): \theta \in \frac{\mathbb{R}}{2 \pi \mathbb{Z}}, t \in \mathbb{R}\right\}$ is precompact in $\mathrm{l}^{2}\left(\langle n\rangle^{2} d n\right)$ after taking Fourier transforms. By monotone convergence, $\forall \epsilon>0$, there exists $\mathrm{R}>0$ such that $\sum_{n \in \mathbb{Z}^{d}, n \geq R}\langle n\rangle^{2}\left|\hat{u}_{+}(n)\right|^{2} \leq \epsilon$. We can conclude $\left\{\left.e^{i \theta} e^{i t \mid}\right|^{2} \widehat{u_{+}}: \theta \in \mathbb{R} / 2 \pi \mathbb{Z}, t \in \mathbb{R}\right\}$ is covered by finitely many balls of radius $O(\epsilon)$, and the claim follows.

Lemma 3 (Diamagnetic inequality) If $u \in \mathrm{H}^{1}\left(\mathbb{T}^{d}\right)$, then $|u| \in \mathrm{H}^{1}\left(\mathbb{T}^{d}\right)$ and

$$
\||u|\|_{H^{1}\left(\mathbb{T}^{d}\right)} \leq\|u\|_{H^{1}\left(\mathbb{T}^{d}\right)} .
$$

Proof. It suffices to verify this when $u$ is smooth. For any $\epsilon>0$, we have

$$
2\left|\sqrt{\epsilon^{2}+|u|^{2}} \nabla \sqrt{\epsilon^{2}+|u|^{2}}\right|=\left|\nabla\left(\epsilon^{2}+|u|^{2}\right)\right|=2|\operatorname{Re}(\bar{u} \nabla u)| \leq 2|u||\nabla u|,
$$

and hence

$\left|\nabla \sqrt{\epsilon^{2}+|u|^{2}}\right| \leq|\nabla u|$. Taking distributional limits as $\epsilon \rightarrow 0$, we obtain the claim.

Lemma 4 ( $\mathrm{H}^{1}$ has no step functions). Let $u \in \mathrm{H}^{1}\left(\mathbb{T}^{d}\right)$ be such that $u(x)$ takes at most two value. Then $\mathrm{u}$ is constant.

Proof. We may assume that $u$ takes 0 and 1 only, thus $u^{2}=u$. On the one hand, differentiating this we obtain $2 u \nabla u=\nabla u$, thus $(1-2 u) \nabla u=0$. On the other hand, since $u^{2}=u,(1-2 u)^{2}=1$, and thus $\nabla u=0$, therefore $u$ is constant.

Proof of Theorem 1. Let $\mathrm{u}_{1} \mathrm{u}_{+}, \theta$ be as above. We may assume $u$ has non-zero mass. From Lemma 2 , we see that $\{\mathrm{u}(\mathrm{t}): \mathrm{t} \in[0, \infty)\}$ is precompact in $H^{1}$. Thus we can find a sequence $\mathrm{t}_{m} \rightarrow \infty$ such that $u\left(\mathrm{t}_{m}\right) \rightarrow v_{0}$ in $\mathrm{H}^{1}\left(\mathbb{T}^{d}\right)$, as $\mathrm{m} \rightarrow \infty$. Applying Lemma 2 and passing to a subsequence, we can also assume $\mathrm{e}^{i t_{m} \Delta} u_{+} \rightarrow v_{+}$in $\mathrm{H}^{1}$, as $\mathrm{m} \rightarrow \infty$. 
Since $u$ has non-zero mass, we see $v_{+}$also has non-zeromass. Let $v^{(m)}: \mathbb{R} \times \mathbb{T}^{d} \rightarrow \mathbb{C}$ be the time-translated solution $v^{(m)}(t)=u\left(t+t_{m}\right)$, thus $v^{(m)}(0) \rightarrow v_{0}$ in $H^{1}\left(\mathbb{T}^{d}\right)$. Let $v: \mathbb{R} \times \mathbb{T}^{d} \rightarrow \mathbb{C}$ be solution to NLS with initial data $v(0)=v_{0}$. By the local well-posedness theory in $H^{1}$ we conclude that $v^{(m)}$ converge uniformly in $H^{1}$ to $v$ on every compact time interval [-T,T].

On the other hand, by hypothesis, $\forall t$ we have

$$
\left\|v^{(m)}(t)-e^{i \theta\left(t+t_{m}\right)} e^{i\left(t+t_{m}\right) \Delta} u_{+}\right\|_{H^{1}} \rightarrow 0 \text {, as } m \rightarrow \infty .
$$

Since $e^{i t_{m} \Delta} u_{+} \rightarrow v_{+}$, as $m \rightarrow \infty$, we conclude that

$$
\left\|v^{(m)}(t)-e^{i \theta\left(t+t_{m}\right)} e^{i t \Delta} v_{+}\right\|_{H^{1}} \rightarrow 0 \text {, as } m \rightarrow \infty .
$$

By taking limits, we conclude that

$$
v(t)=e^{i \alpha(t)} e^{i t \Delta} v_{+}
$$

for some $\alpha(t) \in \mathbb{R} / 2 \pi \mathbb{Z}$.

In particular, since $v_{+}$has non-zero mass, we have

$$
e^{i \alpha(t)}=\frac{1}{\left\|v_{+}\right\|_{L^{2}}^{2}} \int_{\mathbb{T}^{d}} v(t) \overline{e^{i t \Delta} v_{+}} \mathrm{d} x .
$$

From (1) and Sobolev, we see that $v(\mathrm{t})$ is continuously differentiable in $H^{-1}\left(\mathbb{T}^{d}\right)$, and so from the above identity we see that $\alpha$ is continuously differentiable in time. Now we apply i $\partial_{t}+\Delta$ to both sides of (3). Using the NLS equation, we conclude that

$$
\begin{gathered}
\left(\mathrm{i} \partial_{t}+\Delta\right) v=\left(-\mathrm{i} \partial_{t}+\Delta\right)\left(e^{i \alpha(t)} e^{i t \Delta_{v_{+}}}\right) \\
\Rightarrow|v(t, x)|^{p-1} v(t, x)=\alpha^{\prime}(t) e^{i \alpha(t)} e^{i t \Delta_{+}} v_{+}(x) .
\end{gathered}
$$

And thus by (1.3), we have whenever $v(t, x) \neq 0$,

$$
\begin{aligned}
|v(t, x)|^{p-1} e^{i \alpha(t)} e^{i t \Delta} v_{+}(x) & =\alpha^{\prime} e^{i \alpha(t)} e^{i t \Delta} v_{+}(x) \\
\Rightarrow|v(t, x)|^{p-1} & =\alpha^{\prime}(t) .
\end{aligned}
$$

Thus we see that $\forall \mathrm{t},|v(t, x)|$ takes at most two values. By Lemma 3 and Lemma 4 , we conclude that $|v(t, x)|$ is constant in $x$, by (3), we see that the same is true for $\left|e^{i t \Delta} v_{+}(x)\right|$. By mass conservation we conclude that $\left|e^{i t \Delta} v_{+}(x)\right|$ is also constant in time.

Since $v_{+}$has non-zero mass, we can thus write

$$
e^{i t \Delta} v_{+}(x)=A \phi \text {, a.e., }
$$

where $\phi: \mathbb{R} \times \mathbb{T}^{d} \rightarrow \mathbb{S}^{1}$ is some function and $A>0$. Since $v_{+}$was in $H^{1}$, we see that $\phi(t)$ is in $H^{1}$ also for every t. Differentiating the identity $\phi \bar{\phi}=1$, We see that $\partial_{j} \phi \phi$ is imaginary almost everywhere for $j=1,2$, thus $\partial_{j} \phi(t, x)$ is an imaginary multiple of $\phi(t, x)$ for almost every $(t, x)$. This implies the imaginary vector field $\nabla \phi . \bar{\phi}$ is curl-free and thus by Hodge theory, we may write $\nabla \phi . \bar{\phi}=i \nabla \omega$ for some $\omega: \mathbb{R} \times \mathbb{R}^{d} \rightarrow \mathbb{R}$ which is locally in $H^{1}$ uniformly in $t$. This implies that $\nabla\left(\phi e^{-i \omega}\right)=0$, thus by adjusting $\omega$ by a constant independent of space. We may assume $\phi=e^{-i \omega}$, thus $e^{i t \Delta} v_{+}=A e^{i \omega(t, x)}$.

Applying i $\partial_{t}+\Delta$ to both sides, we conclude that

$$
0=\left(\mathrm{i} \partial_{t}+\Delta\right) e^{-i t \Delta} v_{+}
$$




$$
\begin{gathered}
=\left(\mathrm{i} \partial_{t}+\Delta\right)\left(A e^{-i \omega(t, x)}\right) \\
=A\left(e^{i \omega(t, x)} \partial_{t} \omega+\Delta e^{i \omega(t, x)}\right) \\
=A\left(e^{i \omega(t, x)} \partial_{t} \omega+\nabla\left(i \nabla \omega(t, x) \Delta e^{i \omega(t, x)}\right)\right) \\
=A\left(e^{i \omega(t, x)} \partial_{t} \omega+i \Delta \omega(t, x) \Delta e^{i \omega(t, x)}+i \nabla \omega(t, x) i \nabla \omega(t, x) e^{i \omega(t, x)}\right) \\
=A\left(e^{i \omega(t, x)} \partial_{t} \omega+i \Delta \omega(t, x) \Delta e^{i \omega(t, x)}-|\nabla \omega(t, x)|^{2} e^{i \omega(t, x)}\right) \\
=A e^{i \omega(t, x)}\left(\partial_{t} \omega+i \Delta \omega-|\nabla \omega|^{2}\right)
\end{gathered}
$$

in the sense of distributions.

Since A is non-zero, we conclude that

$$
\partial_{t} \omega+i \Delta \omega-|\nabla \omega|^{2}=0
$$

Taking imaginary parts, we conclude that $\Delta \omega=0$ and in particular at time $t$ $=0$, $\omega$ is a harmonic function from $\mathbb{R}^{d}$ to $\mathbb{R}$. On the other hand, from the identity $\nabla \phi \bar{\phi}=i \nabla \omega$, we know that $\nabla \omega$ is periodic, so $\omega$ has at most linear growth. Thus $\omega$ must in fact be linear. Descending back to $\mathbb{T}^{d}$, we conclude that

$$
e^{i \omega(0, x)}=e^{i(n x+\beta)}, \text { for some } n \in \mathbb{Z}^{d}, \beta \in \mathbb{R} / 2 \pi \mathbb{Z} .
$$

Thus, we have

$$
v_{+}(x)=e^{i \omega(0, x)}=e^{i(n x+\beta)}
$$

Since

$$
\begin{aligned}
& e^{i t_{m} \Delta} u_{+} \rightarrow v_{+} \text {in } H^{1} \text {, as } \mathrm{m} \rightarrow \infty, \\
& e^{i t_{m} \Delta} v_{+} \rightarrow u_{+} \text {in } H^{1} \text {, as } \mathrm{m} \rightarrow \infty,
\end{aligned}
$$

but $e^{-i t_{m} \Delta} v_{+}$is a multiple of $A e^{i n x}$ by a phase, thus $u_{+}(x)=\mathrm{A} e^{i r} e^{i n x}$. Applying phase rotation, we may assume $r=0$, thus $u_{+}(x)=A e^{i n x}$, and we have

$$
\left\|u(t)-e^{i \theta(t)} e^{-i t \Delta} \mathrm{A} e^{i n x}\right\|_{H^{1}\left(\mathbb{T}^{d}\right)} \rightarrow 0 \text {, as } t \rightarrow \infty .
$$

From mass and energy conservation, we conclude

$$
\begin{gathered}
\int_{\mathbb{T}^{d}}|u(t, x)|^{2} \mathrm{~d} x=\lim _{t \rightarrow \infty} \int_{\mathbb{T}^{d}}\left|e^{i \theta(t)} e^{-i t \Delta} \mathrm{A} e^{i n x}\right|^{2} \mathrm{~d} x \\
=\lim _{t \rightarrow \infty} \int_{\mathbb{T}^{d}}\left|e^{-i t \Delta} \mathrm{A} e^{i n x}\right|^{2} \mathrm{~d} x \\
=\lim _{t \rightarrow \infty} \int_{\mathbb{T}^{d}}\left|\mathrm{~A} e^{i n x}\right|^{2} \mathrm{~d} x=A^{2}\left|\mathbb{T}^{d}\right|,
\end{gathered}
$$

and

$$
\begin{gathered}
\int_{\mathbb{T}^{d}} \frac{1}{2}|\nabla u(t, x)|^{2}+\frac{1}{p+1}|u(t, x)|^{p+1} \mathrm{~d} x \\
=\lim _{t \rightarrow \infty} \int_{\mathbb{T}^{d}} \frac{1}{2}\left|\nabla\left(e^{i \theta(t)} e^{-i t \Delta} \mathrm{A} e^{i n x}\right)\right|^{2}+\frac{1}{p+1}\left|e^{i \theta(t)} e^{-i t \Delta} \mathrm{Ae}^{i n x}\right|^{p+1} \mathrm{~d} x \\
=\lim _{\mathrm{t} \rightarrow \infty} \int_{\mathbb{T}^{\mathrm{d}}} \frac{1}{2}\left|\nabla\left(\mathrm{A} \mathrm{e}^{\mathrm{inx}}\right)\right|^{2}+\frac{1}{\mathrm{p}+1} \mathrm{~A}^{\mathrm{p}+1}\left|\mathrm{e}^{-\mathrm{it} \Delta} \mathrm{Ae}^{\mathrm{inx}}\right|^{\mathrm{p}+1} \mathrm{dx} \\
=\int_{\mathbb{T}^{d}} \frac{1}{2} A^{2}\left|i n e^{i n x}\right|^{2}+\frac{1}{p+1} A^{p+1} \mathrm{~d} x
\end{gathered}
$$




$$
=\frac{n^{2}}{2} A^{2}\left|\mathbb{T}^{d}\right|+\frac{A^{p+1}}{p+1}\left|\mathbb{T}^{d}\right| .
$$

On the other hand, from Hölder's inequality, we have

$$
\begin{gathered}
\left(\int_{\mathbb{T}^{\mathrm{d}}}|u(t, x)|^{2} d x\right)^{\frac{1}{2}} \leq\|u(t, x)\|_{L_{x}^{p+1}\left(\mathbb{T}^{d}\right)}\left|\mathbb{T}^{d}\right|^{\frac{1}{2}-\frac{1}{p+1}} \\
=\|u(t, x)\|_{L_{x}^{p+1}\left(\mathbb{T}^{d}\right)} \mid \mathbb{T}^{d} \frac{\frac{p-1}{2(p+1)}}{}
\end{gathered}
$$

then

$$
\begin{gathered}
\int_{\mathbb{T}^{d}} \frac{1}{p+1}|u(t, x)|^{p+1} d x \geq \frac{1}{p+1}\left(\int_{\mathbb{T}^{d}}|u(t, x)|^{2} d x\right)^{\frac{p+1}{2}}\left|\mathbb{T}^{d}\right|^{-\frac{p-1}{2}} \\
=\frac{1}{p+1}\left(A^{2}\left|\mathbb{T}^{d}\right|\right)^{\frac{p+1}{2}}\left|\mathbb{T}^{d}\right|^{-\frac{p-1}{2}} \\
=\frac{1}{p+1} A^{p+1}\left|\mathbb{T}^{d}\right| .
\end{gathered}
$$

Thus, we must have

$$
\int_{\mathbb{T}^{d}} \frac{1}{2}|\nabla u(t, x)|^{2} d x=0 .
$$

Thus, $u$ is constant in space, and thus it is of the form

$$
u(t, x)=A e^{i k(t)} .
$$

Applying (1), we see that

$$
\begin{gathered}
\mathrm{i} \partial_{t} u+\Delta u=\left(\mathrm{i} \partial_{t}+\Delta\right) A \mathrm{e}^{i k(t)}=\mathrm{A}\left(k^{\prime}(t) \mathrm{e}^{i k(t)}\right)=\mathrm{A} k^{\prime}(t) e^{i k(t)}|\mathrm{u}|^{\mathrm{p}-1} \mathrm{u} \\
=\mathrm{A}^{\mathrm{p}} \mathrm{e}^{\mathrm{ik}(\mathrm{t})},
\end{gathered}
$$

then

$$
\mathrm{A}^{p}=A k^{\prime}(t) \Rightarrow \mathrm{k}^{\prime}(\mathrm{t})=A^{p-1},
$$

so

$$
k(t)=k(0)+A^{p-1} t,
$$

so $u(t, x)=A \mathrm{e}^{i k(0)} e^{i|A|^{p-1} t}$, which is exactly the form of (2).

\section{References}

[1] Bourgain, J. (1999) Global Well-Posedness of Defocusing 3D Critical NLS in the Radial Case. J. Amer. Math. Soc., 12, 145-171.

https://doi.org/10.1090/S0894-0347-99-00283-0

[2] Cazenave, T. (2003) Semilinear Schrödinger Equations, Courant Lecture Notes in Mathematics, 10. New York University, Courant Institute of Mathematical Sciences, New York, American Mathematical Society, Providence, RI.

[3] Colliander, J., Keel, M., Staffilani, G., Takaoka, H. and Tao, T. (2008) Global Well-Posedness and Scattering for the Energy-Critical Nonlinear Schrödinger Equation in $R^{3}$, Ann. of Math., 167, 767-865.

https://doi.org/10.4007/annals.2008.167.767

[4] Tao, T. (2006) Nonlinear Dispersive Equations: Local and Global Analysis, CBMS Regional Conference Series in Mathematics, 106. American Mathematical Society, Providence, RI. https://doi.org/10.1090/cbms/106 
[5] Colliander, J., Keel, M., Staffilani, G., Takaoka, H. and Tao, T. (2010) Transfer of Energy to High Frequencies in the Cubic Defocusing Nonlinear Schrödinger Equation. Invent. Math, 181, 39-113. https://doi.org/10.1007/s00222-010-0242-2

Submit or recommend next manuscript to SCIRP and we will provide best service for you:

Accepting pre-submission inquiries through Email, Facebook, LinkedIn, Twitter, etc. A wide selection of journals (inclusive of 9 subjects, more than 200 journals)

Providing 24-hour high-quality service

User-friendly online submission system

Fair and swift peer-review system

Efficient typesetting and proofreading procedure

Display of the result of downloads and visits, as well as the number of cited articles Maximum dissemination of your research work

Submit your manuscript at: http://papersubmission.scirp.org/

Or contact jamp@scirp.org 